\title{
The use of multiple respiratory inhalers requiring different inhalation techniques has an adverse effect on COPD outcomes
}

This article was published in the following Dove Press journal:

International Journal of COPD

21 December 2016

Number of times this article has been viewed

\section{Sinthia Bosnic-Anticevich' \\ Henry Chrystyn ${ }^{2}$ \\ Richard W Costello ${ }^{3,4}$ \\ Myrna B Dolovich ${ }^{5}$ \\ Monica J Fletcher ${ }^{6}$ \\ Federico Lavorini ${ }^{7}$ \\ Roberto Rodríguez-Roisin ${ }^{8}$ \\ Dermot Ryan 9,10 \\ Simon Wan Yau Ming ${ }^{2}$ \\ David B Price ${ }^{2,11}$ \\ 'Woolcock Institute of Medical}

Research, School of Medical Sciences, University of Sydney and Sydney Local Health District, Sydney, NSW,

Australia; ${ }^{2}$ Observational and

Pragmatic Research Institute Pte Ltd,

Singapore; ${ }^{3} \mathrm{RCSI}$ Medicine, Royal

College of Surgeons, ${ }^{4}$ RCSI Education

\& Research Centre, Beaumont

Hospital, Beaumont, Dublin, Ireland;

${ }^{5}$ Department of Medicine, Respirology,

McMaster University, ON, Canada;

${ }^{6}$ Education for Health, Warwick, UK; ${ }^{7}$ Department of Experimental and Clinical Medicine, University of Florence, Florence, Italy; ${ }^{8}$ Respiratory Institute, Hospital Clinic, Universitat de Barcelona, Barcelona, Spain;

${ }^{9}$ Optimum Patient Care, Cambridge,

${ }^{10}$ Centre for Population Health

Sciences, University of Edinburgh,

Edinburgh, "Academic Primary Care,

University of Aberdeen, Aberdeen, UK

Correspondence: David B Price Academic Primary Care, Division of Applied Health Sciences, University of Aberdeen, Polwarth Building, Foresterhill, Aberdeen AB25 2ZD, UK

Tel +44 I 223967855

Email dprice@opri.sg
Background: Patients with COPD may be prescribed multiple inhalers as part of their treatment regimen, which require different inhalation techniques. Previous literature has shown that the effectiveness of inhaled treatment can be adversely affected by incorrect inhaler technique. Prescribing a range of device types could worsen this problem, leading to poorer outcomes in COPD patients, but the impact is not yet known.

Aims: To compare clinical outcomes of COPD patients who use devices requiring similar inhalation technique with those who use devices with mixed techniques.

Methods: A matched cohort design was used, with 2 years of data from the Optimum Patient Care Research Database. Matching variables were established from a baseline year of follow-up data, and two cohorts were formed: a "similar-devices cohort" and a "mixed-devices cohort". COPD-related events were recorded during an outcome year of follow-up. The primary outcome measure was an incidence rate ratio (IRR) comparing the rate of exacerbations between study cohorts. A secondary outcome compared average daily use of short-acting beta agonist (SABA).

Results: The final study sample contained 8,225 patients in each cohort (mean age 67 [SD, 10], $57 \%$ males, $37 \%$ current smokers). Patients in the similar-devices cohort had a lower rate of exacerbations compared with those in the mixed-devices cohort (adjusted IRR 0.82, 95\% confidence interval [CI] 0.80-0.84) and were less likely to be in a higher-dose SABA group (adjusted proportional odds ratio $0.54,95 \%$ CI $0.51-0.57$ ).

Conclusion: COPD patients who were prescribed one or more additional inhaler devices requiring similar inhalation techniques to their previous device(s) showed better outcomes than those who were prescribed devices requiring different techniques.

Keywords: chronic obstructive pulmonary disease, inhalation technique, exacerbations, inhaler devices, observational, matched cohort

\section{Introduction}

COPD is a common lung disease characterized by variable day-to-day respiratory symptoms. Management is aimed at both improving symptom control and preventing or reducing exacerbations. ${ }^{1}$ Inhalers deliver medication directly to the lungs and many types of inhaler devices are available. Different devices may require unique inhalation techniques to ensure the medication is delivered optimally. ${ }^{2}$ For example, metered-dose inhalers (MDIs) require a slow and steady inhalation for adequate lung deposition. ${ }^{3,4}$ In contrast, inhalation when using dry-powder inhalers (DPIs) must be quick to disaggregate and dispense the powder and deliver the required dose. ${ }^{4,5}$ Further variations exist, in the dose-loading mechanisms of inhalers (device preparation) and in the resistance to airflow 
within the device. There is also a negative impact of exhaling into the device after priming, which impacts drug delivery for some but not all devices. ${ }^{6}$ With such precision required, it is not surprising that errors in inhalation technique are common and can lead to reduced effectiveness of treatment. ${ }^{7-13}$ Incorrect inhalation technique is well-recognized as a problem with patients using only one inhaler, and it could be compounded when introducing additional inhalers into a treatment regimen. Use of multiple inhalers compared to single inhalers has been shown to have an adverse effect on outcomes, even without consideration of inhalation technique. ${ }^{14,15}$

Despite suggestions that mixing devices could be detrimental to patient outcomes, ${ }^{2}$ no one has yet investigated its real impact in COPD. For such a common illness, with high mortality and significantly impaired quality of life, ${ }^{16,17}$ it is important for research to highlight where problems lie, to allow management guidelines to be addressed accordingly.

This study used real-life observational data to compare and quantify the COPD outcomes for two types of patient those who use multiple inhaler devices with similar inhalation technique and those who use multiple inhaler devices with mixed inhalation techniques.

\section{Materials and methods \\ Data source and permissions}

For this study, data from the Optimum Patient Care Research Database $(\mathrm{OPCRD})^{18}$ - a primary care database containing anonymized, routinely-recorded clinical data from over 2 million patients - were used. The data originated from over 550 General Practices (GPs) across the UK and was supplemented by patient-completed questionnaire data for approximately $10 \%$ of patients with asthma and COPD. The OPCRD has ethical approval from the National Health Service Research Authority to hold and process anonymized research data (Research Ethics Committee reference: 15/EM/0150). This study was conducted to standards recommended for observational research ${ }^{19}$ and was approved by the Anonymized Data Ethics Protocols and Transparency committee - the independent scientific advisory committee for the OPCRD; patient consent was not required due to the retrospective nature of this study, as approved by this committee. This study is registered with the European Network of Centres for Pharmacoepidemiology and Pharmacovigilance. ${ }^{20}$

\section{Study design}

A historical, matched cohort study design was used, creating two study cohorts that differed in terms of the inhaler devices used by patients. The two cohorts were classified after an

additional COPD therapy was prescribed to patients, via a separate inhaler device. Prior to this additional prescription, if patients used more than one device, these multiple devices required similar inhalation technique. After the additional prescription, patients were allocated to either the "similardevices cohort", where patients used two or more inhalers that required similar inhalation technique or the "mixed-devices cohort", where the patients used two or more inhalers, with at least one inhaler requiring a largely different inhalation technique compared to their other device(s). For the main analysis, the inhalers were categorized as "similar" if they were all aerosols (MDI or a soft mist inhaler) or all DPIs. A patient using any combination of these two categories would be allocated to the "mixed-devices" cohort. Hence, a patient using at least one MDI alongside at least one DPI was allocated to the "mixed-devices" category.

To investigate the impact of a more stringent categorization, a sensitivity analysis was also carried out. Here, the DPIs were distinguished further and devices were considered "mixed" if they varied by the level of resistance to airflow through the device or by the number of doses that can be loaded into the device (ie, single dose versus multiple dose). Table 1 shows the specific categorization of devices for each type of analysis.

This study consisted of two continuous years of data, within the period 2008-2015, on either side of an index date.

Table I Device categorization used to form study cohorts

\section{Categorization of devices $^{a}$}

\begin{tabular}{ll}
\hline Main analysis & Sensitivity analysis \\
\hline Aerosols: manual-activated MDI, & $\begin{array}{l}\text { Aerosols: same as in main } \\
\text { manual-activated MDI with spacer, }\end{array}$ \\
analysis
\end{tabular}

breath-actuated MDI and soft

mist inhaler (pMDIs, CFC-free

inhalers, breath-actuated inhalers,

Clenil modulite, pMDIs + pacer,

Autohaler, Easibreathe, Respimat)
DPIs: single-dose capsule DPI

(Breezhaler, Aerolizer, Handihaler),

multi-dose DPI with medium

resistance (Accuhaler [Diskus],

Novolizer, Genuair, Elipta) and

multi-dose DPI with medium-high

or high resistance (Turbohaler,

Clickhaler, Pulvinal, Twisthaler,

Easyhaler, Duoresp)

\section{Single-dose capsule DPI \\ (Breezhaler, Aerolizer, \\ Handihaler) \\ Multi-dose DPI with medium resistance: (Accuhaler [Diskus], Novolizer, Genuair, Ellipta) \\ Multi-dose DPI with medium-high or high resistance (Turbohaler, Clickhaler, Pulvinal, Twisthaler, Easyhaler, Duoresp)}

Note: ${ }^{2}$ Devices from within categories (identified with headings in bold) are considered "similar" and devices from more than one category are considered "mixed".

Abbreviations: DPI, dry-powder inhaler; MDI, metered dose inhaler; PMDI, pressurized metered dose inhaler. 
The index date was identified as the point at which a prescription of additional COPD therapy was made, which required the use of an inhaler device separate from the current COPD treatment of that patient. The year preceding this date was the baseline year and the subsequent year was the outcome year. Study cohorts ("similar" versus "mixed") were matched in a ratio of 1:1 to minimize differences that could affect the comparison of study outcomes. Matching variables were identified through comparison of baseline variables between unmatched cohorts and by assessing the variables that were most predictive of COPD outcome (number of exacerbations).

\section{Inclusion and exclusion criteria}

Patient records were subject to the following inclusion criteria: record of diagnosis of COPD; at least 40 years of age at the date of initial prescription; an initial prescription of COPD therapy, including short-acting beta agonist (SABA), short-acting muscarinic agonist (SAMA), long-acting beta agonist (LABA) or long-acting muscarinic agonist, which could be given alone or in combination, but must be given in "similar" devices if in combination; one or more prescriptions of additional COPD therapy via a separate inhaler device, on the index date or during the outcome year (a change in inhaler device, rather than the addition of a device, does not fall under the inclusion criteria); at least 2 years of continuous medical records from GP (at least 1 year of which is when patient is on initial therapy and 1 year on additional therapy).

Patients were excluded if they had "mixed" devices prior to the index date or if they had at least one prescription for nebulizer prior to the index date, as this is another category of device altogether.

\section{Outcome measures}

The primary outcome was the rate of moderate/severe COPD exacerbations $^{21}$ during the outcome year, comparing the similar-devices cohort to the mixed-devices cohort. Details of exacerbation criteria are summarized in the notes of Table 2. The comparison of exacerbation rates was done by estimating an adjusted incidence rate ratio (IRR) - adjusted by factors that were not used in the matching process but were found to be different between cohorts at baseline. A secondary outcome was the average daily SABA use during the outcome year, which was compared between study cohorts. A proportional odds ratio (OR) was estimated, comparing the odds of being in higher SABA dose categories. Again, this was adjusted by important factors that differed at baseline.
Table 2 Baseline demographic characteristics and COPD severity of study cohorts

\begin{tabular}{|c|c|c|}
\hline Patient characteristics & $\begin{array}{l}\text { Similar-devices } \\
\text { cohort }(n=8,225)\end{array}$ & $\begin{array}{l}\text { Mixed-devices } \\
\text { cohort }(n=8,225)\end{array}$ \\
\hline \multicolumn{3}{|c|}{ Demographic characteristics } \\
\hline \multicolumn{3}{|l|}{ Age (years) } \\
\hline $40-60, \mathrm{n}(\%)$ & $1,856(22.6)$ & $\mathrm{I}, 856(22.6)$ \\
\hline $61-80, \mathrm{n}(\%)$ & $5,863(71.3)$ & $5,863(71.3)$ \\
\hline$>80, \mathrm{n}(\%)$ & $506(6.2)$ & $506(6.2)$ \\
\hline Mean (SD) & $67.3(9.9)$ & $67.2(9.7)$ \\
\hline Male, n (\%) & $4,645(56.5)$ & $4,645(56.5)$ \\
\hline \multicolumn{3}{|l|}{ Body mass index $\left(\mathrm{kg} / \mathrm{m}^{2}\right)$} \\
\hline$<18.5, \mathrm{n}(\%)$ & I5I (I.8) & $15 \mid(\mid .8)$ \\
\hline I8.5-24.9, n (\%) & $2,892(35.2)$ & $2,892(35.2)$ \\
\hline 25-29.9, n (\%) & $2,98 \mathrm{I}(36.2)$ & $2,98 I(36.2)$ \\
\hline$\geq 30, \mathrm{n}(\%)$ & $2,20 \mathrm{I}(26.8)$ & $2,20 \mathrm{I}(26.8)$ \\
\hline Mean (SD) & $27.4(6.1)$ & $27.4(5.7)$ \\
\hline \multicolumn{3}{|l|}{$\begin{array}{l}\text { Smoking status (closest } \\
\text { to index date) }\end{array}$} \\
\hline Nonsmoker, n (\%) & $584(7.1)$ & $584(7.1)$ \\
\hline Current smoker, $\mathrm{n}(\%)$ & $3,029(36.8)$ & $3,029(36.8)$ \\
\hline Ex-smoker, n (\%) & $4,612(56.1)$ & $4,612(56.1)$ \\
\hline \multicolumn{3}{|l|}{ COPD severity } \\
\hline \multicolumn{3}{|l|}{$\mathrm{FEV}_{1} \%$ predicted $^{\mathrm{a}}$} \\
\hline $\begin{array}{l}\text { GOLD I: } \mathrm{FEV}_{1} \geq 80 \\
\text { (mild), } \mathrm{n}(\%)\end{array}$ & $717(8.7)$ & $717(8.7)$ \\
\hline $\begin{array}{l}\text { GOLD 2: } 50 \leq \mathrm{FEV}_{1}<80 \\
\text { (moderate), } \mathrm{n}(\%)\end{array}$ & $4,659(56.6)$ & $4,659(56.6)$ \\
\hline $\begin{array}{l}\text { GOLD 3: } 30 \leq \mathrm{FEV}_{1}<50 \\
\text { (severe), } \mathrm{n}(\%)\end{array}$ & $2,003(24.4)$ & $2,003(24.4)$ \\
\hline $\begin{array}{l}\text { GOLD 4: } \mathrm{FEV},<30 \\
\text { (very severe), } \mathrm{n}(\%)\end{array}$ & $846(10.3)$ & $846(10.3)$ \\
\hline Mean (SD) & $55.9(22.6)$ & $55.7(22.5)$ \\
\hline \multicolumn{3}{|l|}{ COPD exacerbations ${ }^{\mathrm{b}}$} \\
\hline $0, \mathrm{n}(\%)$ & $4,226(51.4)$ & $4,226(51.4)$ \\
\hline $\mathrm{I}, \mathrm{n}(\%)$ & $2,394(29.1)$ & $2,394(29.1)$ \\
\hline $2, \mathrm{n}(\%)$ & $972(11.8)$ & $972(11.8)$ \\
\hline$\geq 3, \mathrm{n}(\%)$ & $633(7.7)$ & $633(7.7)$ \\
\hline
\end{tabular}

Notes: Variables were not compared statistically as all were used in matching process. ${ }^{2} \mathrm{FEV}, \mathrm{\%}$ predicted grouped by GOLD classifications; ${ }^{\mathrm{b}}$ moderate/severe exacerbations were identified by acute course of oral corticosteroids (OCS) unlikely to be maintenance therapy, antibiotics prescribed within a lower respiratory consultation or admission to hospital/emergency department for COPD or following lower respiratory consultation. Further details of prescriptions of OCS and antibiotics are found in Table 3.

Abbreviations: SD, standard deviation; $\mathrm{FEV}_{1}$, forced expiratory volume in I second; GOLD, Global initiative for chronic Obstructive Lung Disease.

\section{Statistical analysis}

Summary statistics were computed for baseline characteristics of study cohorts, including demographic variables, COPD-related characteristics and therapies, comorbidities and co-medications. Variables found to be significantly different between cohorts $(P<0.05)$ were potentially used as adjusting variables for the primary and secondary analyses. If these variables were found to be unimportant in the multivariate models for the primary/secondary outcomes, they 
were removed to arrive at the most parsimonious model. Confounders were checked for co-linearity using Spearman's correlation coefficients for linear relationships, and plots and univariate logistic regression models for non-linear relationships. If colinearity was observed between several adjusting variables in the primary and secondary multivariate models, the most significant of the variables was retained in the model. Through this process, all available adjusting variables that impacted the unadjusted results were included. Summary statistics were also computed for COPD outcomes, that is the number of exacerbations and average daily SABA use.

The primary analysis fitted a negative binomial regression model to estimate the IRR for rate of exacerbations, with 95\% confidence intervals (CIs). A conditional, fixed effects version of this model was applied to adjust for confounders and account for overdispersion in the data.

For the secondary analysis, average daily SABA use during the outcome year was calculated as follows:

$\frac{\text { Number of inhalers } * \text { doses per inhaler }}{365} \times$ dosage $(\mu \mathrm{g})$

This was grouped into five categories with cutoff points chosen using quintiles. This strengthened the model used to investigate the secondary outcome by ensuring adequate numbers of patients in each category. The model used was an ordinal logistic regression model to estimate the proportional OR (with 95\% CI) of being prescribed a higher average daily SABA drug dosage, comparing the similar-devices cohort to the mixed-devices cohort. Standard errors were adjusted by appropriate confounders.

The aforementioned primary and secondary analyses were repeated for the sensitivity analysis mentioned previously, where the matched cohorts were based on an alternative categorization of devices. A further sensitivity analysis was carried out on a subgroup of the study sample, as determined by the COPD treatment received during the outcome year. Patients were categorized according to the combination of therapy received, for example, $\mathrm{LABA} \pm \mathrm{SAMA} \pm$ $\mathrm{SABA}$, and inhaled corticosteroids (ICS) $\pm \mathrm{SAMA} \pm \mathrm{SABA}$. The largest group was analyzed separately to investigate whether the effect found in the main analysis remained in this subgroup.

The level of statistical significance was set at 5\% (ie, $P<0.05$ ). No prospective power calculation was carried out since the sample size was determined by the number of eligible patients in the OPCRD according to the inclusion criteria and by the number who could then be matched exactly to a patient in the opposite cohort. All categorization and coding of variables, and definition of COPD-related variables, were approved by the steering committee. Analyses were carried out using IBM SPSS Statistics version 22 and Stata version 14.

\section{Results}

\section{Patients}

After applying inclusion and exclusion criteria, 71,517 patients with COPD were identified from the OPCRD. This number was reduced to 52,378 following removal of duplicates and of records with missing observations for the matching variables. The chosen matching variables were age, sex, body mass index (BMI), smoking status, forced expiratory volume in 1 second $\left(\mathrm{FEV}_{1}\right) \%$ predicted, number of moderate/severe exacerbations during baseline year, number of types of COPD treatment, having active asthma and index year. After 1:1 matching, there were 8,225 patients in each study cohort (Figure 1). For the alternative categorization of devices, (Table 1) there were 7,545 patients in each matched cohort.

Patients in both cohorts had a mean age of 67 years $(\mathrm{SD} \approx 10)$, with the majority $(71.3 \%)$ aged between 61 and 80 years (Table 2 ). Just over half were males (56.5\%), mean BMI was 27.4 ( $\mathrm{SD} \approx 6$ ) and $36.8 \%$ were current smokers at the time of index date. In the baseline year, mean $\mathrm{FEV}_{1} \%$ predicted was $\sim 56(\mathrm{SD} \approx 23)$ and $51.4 \%$ of patients had zero moderate or severe exacerbations, while $7.7 \%$ had 3 or more exacerbations.

During the baseline year, $17.5 \%$ of patients in the similardevices cohort and $18.9 \%$ in the mixed-devices cohort required one course of acute oral corticosteroids (OCS) (Table 3). Equivalent percentages for at least one course of antibiotics with a lower respiratory consultation were $21.9 \%$ and $21.3 \%$. Over half of patients in each cohort required at least one COPD-related consultation. The majority of patients in each cohort were receiving only one medication type of COPD treatment (53.2\%), and $40 \%$ were receiving two types. Here, medication type refers to the type of COPD therapy, whether inhaled (eg, SABA and ICS) or in tablet form (eg, theophylline and mucolytes). Comparison between cohorts showed statistically significant differences, in that more patients in the mixed-devices cohort needed; at least one acute OCS course; three or more antibiotics courses; higher SABA inhaler usage per day and at least one COPD-related consultation, during the baseline year. Due to these significant differences, which could be interpreted as an increased likelihood of exacerbation in the mixed-devices cohort, acute 


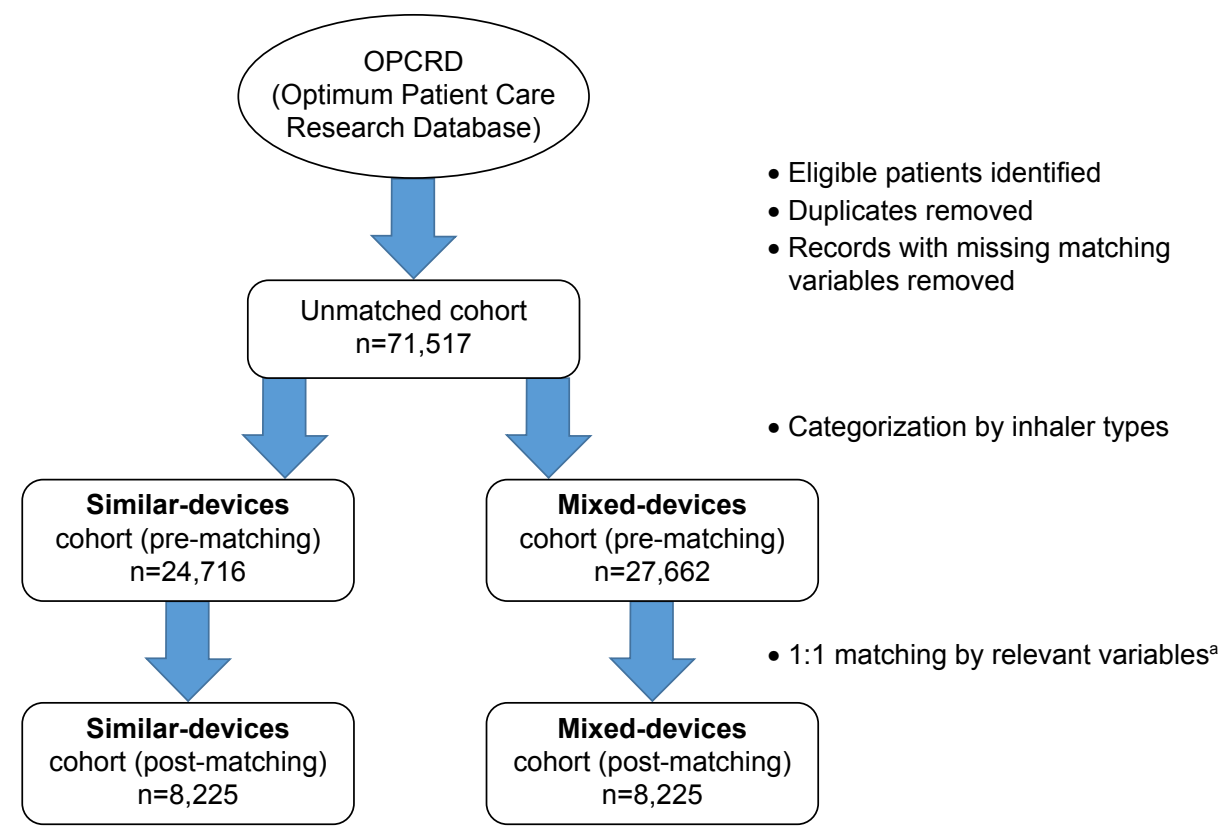

Figure I Flow diagram showing study cohorts derived from Optimum Patient Care Research Database.

Notes: aMatching variables included are as follows: age, sex, body mass index, smoking status, forced expiratory volume in I second \% predicted, number of moderate/ severe exacerbations during baseline year, number of types of COPD treatment, active asthma and index year. Matching variables were derived from exploratory analysis of I year of baseline data.

course of OCS, course of antibiotics, SABA use and COPDrelated consultations were investigated as potential adjusting variables in the primary outcome model.

Information about patient comorbidities, baseline comedications and other measures of COPD severity is given in Table S1. Significant differences (in percent) between matched cohorts were found in patients with rhinitis (less in mixed-devices cohort), eczema (less in mixed-devices cohorts), osteoporosis (more in mixed-devices cohorts) and pneumonia (more in mixed-devices cohorts). These comorbidities, as well as statin-taking (more prevalent in mixed-devices cohort), were investigated as potential adjusting variables in the main analysis. Significant differences were also observed for modified Medical Research Council dyspnea scale score and Global initiative for chronic Obstructive Lung Disease classification, but neither of these was used as a matching or adjusting variable due to the number of missing observations. Specific combinations of COPD treatment during the baseline year are given in Table S2.

\section{Primary outcome}

The number of moderate/severe exacerbations during the outcome year declined in both cohorts with respect to the baseline year, with $58.5 \%$ of patients in the similar-devices cohort and $53.4 \%$ in the mixed-devices cohort, having zero exacerbations in the outcome year (Table 4). After adjustment by confounders, including course of antibiotics, asthma diagnosis and paracetamol use, the rate of exacerbations was lower in the similar-devices cohort compared with the mixed-devices cohort, with an IRR of 0.82 (95\% CI 0.80-0.84; Figure 2).

\section{Secondary outcome}

During the outcome year, $18.9 \%$ of patients in the similardevices cohort and $26.4 \%$ in the mixed-devices cohort used $>550 \mu \mathrm{g}$ SABA per day (Table 4). In general, patients in the similar-devices cohort were less likely to be in a higher SABA dose group compared to mixed-devices cohort, with an adjusted proportional OR of 0.54 (95\% CI $0.51-0.57$; Figure 2).

\section{Sensitivity analyses}

The first sensitivity analysis was carried out on study cohorts that were based on an alternative categorization of inhaler devices - one that included differences in DPIs $(n=7,545$ in each cohort). The similar-devices cohort based on DPI use was further categorized into DPIs which were single-dose capsule products, DPIs with medium resistance and DPIs with medium to high and high resistance (as classified by Laube et $\mathrm{al}^{4}$ ). Results for the primary outcome were similar to the main analysis, with the similar-devices cohort showing a reduced rate of moderate/severe exacerbations compared to the mixed-devices cohort (IRR 0.82 , 95\% CI 0.78-0.86; 
Table 3 Baseline COPD-related therapy of study cohorts

\begin{tabular}{|c|c|c|c|}
\hline \multirow{2}{*}{$\begin{array}{l}\text { COPD-related } \\
\text { therapies }\end{array}$} & \multicolumn{2}{|l|}{ n (\%) } & \multirow[t]{2}{*}{$P$-value } \\
\hline & $\begin{array}{l}\text { Similar- } \\
\text { devices cohort } \\
(n=8,225)\end{array}$ & $\begin{array}{l}\text { Mixed-devices } \\
\text { cohort } \\
(n=8,225)\end{array}$ & \\
\hline $\begin{array}{l}\text { Acute oral } \\
\text { corticosteroid (OCS) } \\
\text { courses }^{b}\end{array}$ & & & 0.019 \\
\hline 0 & $6,010(73.1)$ & $5,882(71.5)$ & \\
\hline 1 & I,437 (I7.5) & I,557 (I8.9) & \\
\hline 2 & $489(6.0)$ & 487 (5.9) & \\
\hline$\geq 3$ & $289(3.5)$ & $299(3.6)$ & \\
\hline $\begin{array}{l}\text { Antibiotic courses with } \\
\text { a lower respiratory } \\
\text { consultation }^{c}\end{array}$ & & & 0.031 \\
\hline 0 & $5,111(62.1)$ & $5,205(63.3)$ & \\
\hline I & I,803 (21.9) & I,755 (2।.3) & \\
\hline 2 & 782 (9.5) & 725 (8.8) & \\
\hline$\geq 3$ & $529(6.4)$ & $540(6.6)$ & \\
\hline $\begin{array}{l}\text { Number of types of } \\
\text { COPD treatment }\end{array}$ & & & NA \\
\hline I & $4,374(53.2)$ & $4,374(53.2)$ & \\
\hline 2 & $3,289(40.0)$ & $3,289(40.0)$ & \\
\hline 3 & $545(6.6)$ & $545(6.6)$ & \\
\hline 4 & $17(0.2)$ & $17(0.2)$ & \\
\hline $\begin{array}{l}\text { SABA inhaler usage } \\
\text { ( } \mu \text { g per day) }\end{array}$ & & & $<0.00 \mathrm{I}$ \\
\hline 0 & 735 (8.9) & $839(10.2)$ & \\
\hline $\mathrm{I}-55$ & $2,174(26.4)$ & I,896 (23.1) & \\
\hline $55-165$ & I,927 (23.4) & I,959 (23.8) & \\
\hline $165-440$ & I,560 (19.0) & I,588 (19.3) & \\
\hline$>440$ & I,829 (22.2) & $1,943(23.6)$ & \\
\hline $\begin{array}{l}\text { COPD-related } \\
\text { consultations }\end{array}$ & & & $<0.00$ I \\
\hline 0 & $3,532(42.9)$ & $3,257(39.6)$ & \\
\hline I & $2,476(30.1)$ & $2,501(30.4)$ & \\
\hline$\geq 2$ & $2,217(27.0)$ & $2,467(30.0)$ & \\
\hline
\end{tabular}

Notes: ${ }^{a}$-values are from conditional logistic regression models. ${ }^{b}$ All acute OCS courses that are definitely not maintenance therapy and/or all courses where dosing instructions suggest exacerbation treatment (eg, a reducing dose or a fixed term specified) and/or all courses with no dosing instructions, but unlikely to be maintenance therapy due to prescription strength or frequency of prescriptions. 'Lower respiratory consultation refers to lower respiratory diagnostic codes (including asthma, COPD and LRTI Read codes), or asthma/COPD review codes excluding any monitoring letter codes, or lung function and/or asthma monitoring, and any additional respiratory examinations, referrals, chest X-rays, or events. $b, c$ Where $>$ I OCS course/antibiotic prescription occurred within 2 weeks of each other, these events were considered to be the result of the same course. NA indicates that no comparison was done as variable was used for matching. Abbreviations: LRTI, lower respiratory tract infection; SABA, short-acting beta agonist.

Figure S1). Results for the secondary analysis showed the same effect as in the main analysis, but with slightly weaker magnitude: similar-devices cohort was less likely to be in a higher category of average SABA dose per day during the outcome year (OR 0.61, 95\% CI 0.57-0.64).

The second sensitivity analysis was applied to the largest subgroup of COPD treatment combination during the
Table 4 COPD outcomes in each study cohort during the outcome year

\begin{tabular}{lll}
\hline COPD outcome & $\mathrm{n}(\%)$ & \\
\cline { 2 - 3 } & $\begin{array}{l}\text { Similar-devices } \\
\text { cohort }(\mathrm{n}=\mathbf{8 , 2 2 5})\end{array}$ & $\begin{array}{l}\text { Mixed-devices } \\
\text { cohort }(\mathrm{n}=\mathbf{8 , 2 2 5})\end{array}$ \\
\hline
\end{tabular}

\begin{tabular}{lll}
\hline $\begin{array}{l}\text { Number of } \\
\text { exacerbations }\end{array}$ & \\
0 & $4,810(58.5)$ & $4,389(53.4)$ \\
I & $1,985(24.1)$ & $2,004(24.4)$ \\
2 & $828(10.1)$ & $959(11.7)$ \\
$3+$ & $602(7.3)$ & $873(10.6)$ \\
Average daily & & \\
SABA dosage ${ }^{a}(\mu g)$ & & \\
0 & $1,437(17.5)$ & $524(6.4)$ \\
$<I I 0$ & $1,742(21.2)$ & $1,698(20.6)$ \\
III-300 & $1,706(20.7)$ & $1,847(22.5)$ \\
$30 I-550$ & $1,788(21.7)$ & $1,984(24.1)$ \\
$>550$ & $1,552(18.9)$ & $2,172(26.4)$ \\
\hline
\end{tabular}

Notes: average daily SABA dosage grouped by quintiles, according to similardevices cohort.

Abbreviation: SABA, short-acting beta agonist.

outcome year, which was ICS $\pm \mathrm{LABA} \pm \mathrm{SAMA} \pm \mathrm{SABA}$ (Table S3). This subgroup consisted of 3,680 patients in the similar-devices cohort (44.7\% of the total patients) and 2,859 patients in the mixed-devices cohort (34.8\% of the total patients). Again, the rate of moderate/severe exacerbations was reduced in the similar-devices cohort compared with the mixed-devices cohort but to a lesser extent than in the main analysis (IRR $0.90,95 \%$ CI $0.82-0.99$; Figure S2). There was also a weaker effect than the main analysis on odds of being in a higher group of daily SABA dose, though it was still significant in favor of similar-devices cohort (OR 0.70, 95\% CI 0.64-0.76).

Combining both sensitivity analyses, that is, the largest treatment combination subgroup after the alternative categorization of devices, led to similar results (Figure S3).

\section{Discussion}

This study demonstrates that patients with COPD who were prescribed inhalers requiring similar inhalation technique to the inhalers they were already using had better COPD outcomes than patients whose additional inhalers required a different technique. Specifically, patients using similar devices had a lower rate of exacerbations and were less likely to use higher doses of SABA medication, suggesting better symptom control. This effect was present after matching/ adjustment of important patient characteristics and common predictors of future exacerbations.

There is a wide range of inhalers for COPD on the market, and no pharmaceutical company manufactures all types 


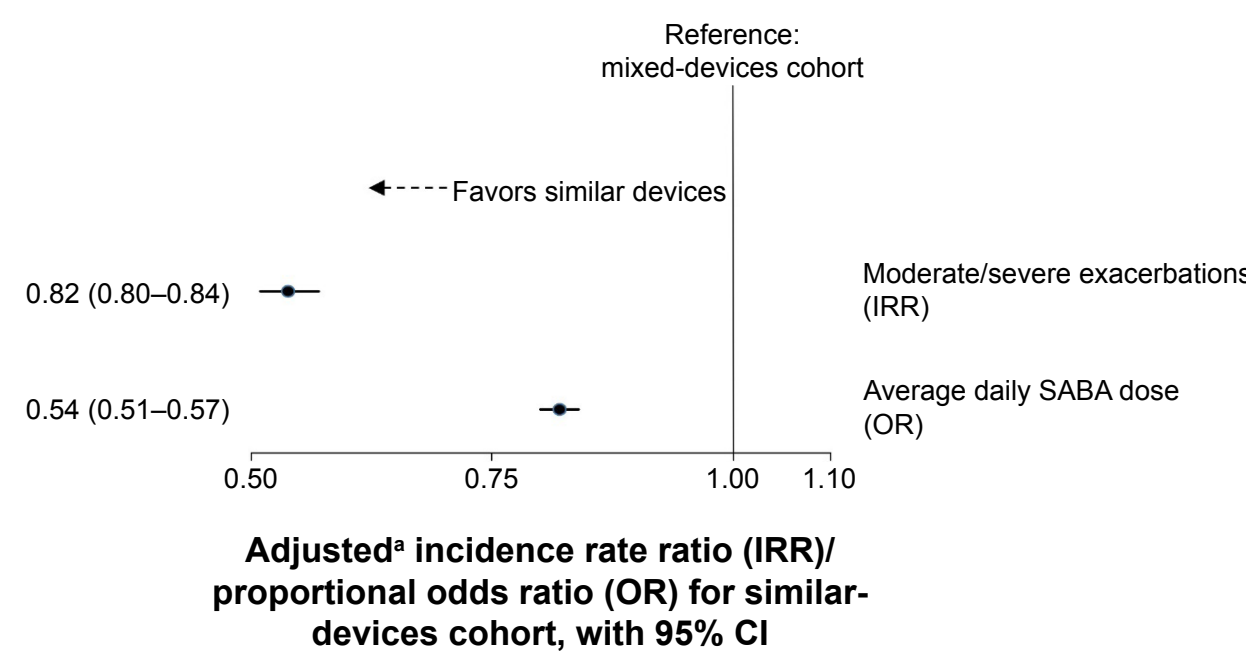

Figure 2 Effect of similar versus mixed devices on primary and secondary COPD outcomes.

Notes: aIRR adjusted by antibiotic course, asthma diagnosis and paracetamol use. OR adjusted by baseline SABA and osteoporosis.

Abbreviations: $\mathrm{Cl}$, confidence interval; SABA, short-acting beta agonist.

of available inhaled treatment. This leads to inhaler devices requiring different inhalation techniques. Previous literature has hypothesized that mixing inhaler devices could cause confusion and have an adverse effect on patient outcomes in COPD and asthma. ${ }^{2}$ The main finding of the current study is consistent with this hypothesis and with a recent asthma study that had a similar research question comparing patients whose reliever and controller devices were both breath-actuated inhalers (BAIs) to patients with BAI as controller and MDI as reliever. ${ }^{22}$ They found that the patients with only BAIs were more likely to achieve asthma control during 1 year of follow-up than the patients with different inhalers. The current results are also consistent with studies showing that simpler treatment regimens are more effective in COPD. Large observational studies by Yu et a ${ }^{14}$ and Chrischilles et al ${ }^{15}$ found that patients using multiple inhaler devices, rather than a single device, had increased hospital resource utilization and number of exacerbations. Although the cohorts in the current study were comparable in terms of number of devices, the trend toward simplicity is common in both scenarios.

The most likely reason for reduced effectiveness in patients with mixed devices is that the variation in inhalation technique leads to inhalation errors. Suboptimal inhalation results in medication not being delivered to the target area adequately. In a study involving asthma and COPD patients, inhaler errors were associated with several adverse outcomes, including hospitalization. ${ }^{23}$ Errors may be more common in specific types of patient. For example, MDIs require good coordination to ensure actuation coincides with (slow) inhalation. In contrast, although DPIs are breath-actuated, they require rapid and forceful inhalation. Additionally, errors have been shown to increase with age..$^{23,24}$ As respiratory function is reduced in older people, this may impact the ability to effectively use a DPI. MDI use may also be affected with increasing age due to reduced hand strength to correctly push the inhaler. However, cohorts in the current study were well matched in terms of age and comorbidity, so it is likely that common errors would be present in both cohorts, whether devices were mixed or not. Instead, a possible explanation to the observed difference between mixed-devices and similardevices cohorts is that as errors are common when using one type of device, there may be even more errors when there are several types of device in use.

The main analysis suggested that patients using all MDI or all DPI devices have better outcomes; the study then went further by considering specific types of DPI. This distinction may be more meaningful from the patient perspective, particularly with regard to dose preparation. For example, multidose DPIs have a mechanism that must be rotated to load the medication and so is an additional method of use that must be learned even if the patient is familiar with single-dose DPIs. The current study results showed that the impact on COPD outcomes for this alternative categorization of devices was similar to the main analysis. This suggests that the essential differences between types of DPI may be as important as the more obvious differences between MDIs and DPIs and could act as a barrier to correct inhaler use. Differences in general could also act as a barrier to patient adherence, in turn reducing effectiveness. More complex treatment regimens may be off-putting to patients. For example, a study by $\mathrm{Yu}$ et $\mathrm{a}^{25}$ found that patients using multiple inhalers were less likely to be adherent than those using a single inhaler. 
The current study observed an $18 \%$ reduction in exacerbation rate when inhalers with similar techniques were used and observed that $41 \%-45 \%$ of patients in either cohort had at least one moderate/severe exacerbation during the outcome year. This $18 \%$ reduction, even if modest, is important in relieving the burden on the COPD patient population. Further, it will have an impact on the high health care costs. ${ }^{26}$

The strength of this study is that it analyzed a large sample from a high-quality database, representative of the real-life COPD patient population. Randomized controlled trials (RCTs) often study a highly selected patient sample and the results cannot necessarily be extrapolated to the general patient population; this makes observational studies an essential contribution to evidence. ${ }^{27}$ Further, in contrast to RCTs where the length of follow-up is often limited, this study obtained 1-year follow-up data, which could capture relatively infrequent events such as exacerbations. Likewise, baseline characteristics were not based on a cross-sectional view from a specified date, but on 1 year of baseline data from multiple index dates to form comparable cohorts.

We addressed the main drawback of an observational study, such as lack of randomization, by applying population matching to the study design and adjustment for confounders to analyses. This was especially important as the baseline comparisons showed that patients in the mixed-devices cohort (though the devices were not mixed during the baseline period) appeared to be less controlled than those in the similar-devices cohort. Without allowing for these initial differences, the comparison of outcomes would be biased. To allow this, confounding was minimized as much as possible by adjusting statistical models by factors such as COPD severity and comorbidities. This was also important as the study sample, by definition, were patients whose symptoms were increasing to the point where additional treatment was required (ie, the index date). Number of treatment types was also used as a matching variable; an imbalance here could have caused bias in terms of adherence. Relevant sensitivity analyses were carried out to test the robustness of the main results. This included subgroup analysis in the largest treatment combination group, which confirmed that the main effect was not being driven by less common treatment combinations.

Limitations of the current study include the possibility of residual confounding, which may be present despite the measures described earlier. An unmeasured confounder that may have introduced bias is any cognitive disorder in the patients. This is relevant in an intervention that depends on knowing how to use multiple devices. However, this bias will have been reduced somewhat when allowing for age and co-medications. This study did not take into account specific types of medication, which may be a source of bias. Salbutamol, for example, is used frequently in reliever MDIs, whereas controller medications are more diverse. This is something to be considered in future studies.

This study did not capture information about patient adherence or about specific errors in inhaler use. Such data may have offered a useful insight, as these factors may drive the effect on outcomes. It was considered outside the scope of the primary research question in this study; however, it may be of interest to investigate adherence in future studies, especially with evidence suggesting variation in level of adherence between types of device. ${ }^{28}$ A further limitation of this study is that patients were not distinguished within cohorts by their device type, that is, the proportion of patients in the similar-devices cohort using only DPIs or only MDIs is unknown. This may have led to differences between cohorts if it was the case that the mixed-devices cohort had a much larger proportion of DPI users than the similardevices cohort. Differences could arise due to variation in adherence levels or inhaler errors made with increasing age, as mentioned previously.

There have already been calls for more careful consideration to be given to the choice of prescribed inhaler devices in COPD. ${ }^{29}$ The Aerosol Drug Management Improvement Team suggested a strategy in 2011, ${ }^{29}$ which offered physicians guidelines for various aspects of COPD management, including choice of inhaler. A previous study found that physicians usually base inhaler choices on the age, skills and physical capacity of their patients. ${ }^{30}$ The current study provides evidence to suggest that prescribing inhalers that require similar inhalation technique should be a further consideration when choosing additional therapies.

\section{Conclusion}

After being prescribed one or more additional inhaler devices, COPD patients who used multiple devices that required similar inhalation techniques had more favorable clinical outcomes in comparison with patients who used devices that required mixed inhalation techniques. This relationship existed after controlling for likely confounders, suggesting that mixing devices may inherently lead to incorrect use and/or reduced patient adherence. This is an intuitive result, consistent with numerous sources in the literature. However, similar studies on different data sets would be useful in order to consolidate evidence and support recommendations in primary care management. 


\section{Acknowledgments}

This study was funded by Teva Pharmaceutical Industries Ltd. The authors would like to thank Lisa Law and Simon van Rysewyck for medical writing and Arjun Jain for statistical analysis. Many thanks to Derek Skinner for preparation of data for analysis.

\section{Author contributions}

All authors contributed toward data analysis, drafting and critically revising the paper and agree to be accountable for all aspects of the work.

\section{Disclosure}

SBA has no shares in any pharmaceutical companies. She has received sponsorship to carry out studies, together with Board Membership, consultant agreements and honoraria for presentation, from several pharmaceutical companies that market inhaled products. These include AstraZeneca, GlaxoSmithKline, Meda, Mundipharma and Teva.

$\mathrm{HC}$ has no shares in any pharmaceutical companies. He has received sponsorship to carry out studies, together with Board Membership, consultant agreements and honoraria for presentation, from several pharmaceutical companies that market inhaled products. These include Almirall, AstraZeneca, Boehringer Ingelheim, Chiesi, GlaxoSmithKline, Innovata Biomed, Meda, Napp Pharmaceuticals, Mundipharma, NorPharma, Norvartis, Orion, Sanofi, Teva, Truddell Medical International, UCB and Zentiva. Research sponsorship has also been received from grant awarding bodies (EPSRC and MRC). He is the owner of Inhalation Consultancy Ltd. He is also an employee of Research in Real Life Ltd, which conducted this study and which has conducted paid research in respiratory disease on behalf of the following other organizations in the past 5 years: Aerocrine, AKL Ltd, Almirall, AstraZeneca, Boehringer Ingelheim, Chiesi, GlaxoSmithKline, Meda, Mundipharma, Napp, Novartis, Orion, Takeda, Teva, and Zentiva, a Sanofi company.

$\mathrm{RC}$ has a patent on an audio-based technology to assess response to inhaled medication. He currently has grants from GSK and Aerogen and receives speaker and Advertisement board fees from GSK Novartis, Boehringer and TEVA.

FL has no shares in any pharmaceutical companies. FL reports personal fees from AstraZeneca, Boehringer Ingelheim, Chiesi, Cipla and Teva Pharmaceuticals. FL has board membership with Boehringer Ingelheim, Chiesi, Teva Pharmaceuticals. RRR reports grants and personal fees from Almirall, AstraZeneca, Boehringer Ingelheim, Ferrer Group,
Mylan, Novartis, Pearl Therapeutics, Takeda, and TEVA and grants and personal fees from Menarini during the conduct of the study. He is member of the GOLD Board of Directors and of the Scientific Committee.

SY is employed by Observational and Pragmatic Research Institute Pte Ltd, which receives funding from UK National Health Service, British Lung Foundation, Aerocrine, AKL Ltd, Almirall, AstraZeneca, Boehringer Ingelheim, Chiesi, Eli Lilly, GlaxoSmithKline, Meda, Merck, Mundipharma, Napp, Novartis, Orion, Pfizer, Respiratory Effectiveness Group, Takeda, Teva Pharmaceuticals and Zentiva.

DP has board membership with Aerocrine, Almirall, Amgen, AstraZeneca, Boehringer Ingelheim, Chiesi, Meda, Mundipharma, Napp, Novartis and Teva Pharmaceuticals; consultancy with Almirall, Amgen, AstraZeneca, Boehringer Ingelheim, Chiesi, GlaxoSmithKline, Meda, Mundipharma, Napp, Novartis, Pfizer and Teva Pharmaceuticals; grants and unrestricted funding for investigator-initiated studies (conducted through Research in Real-Life Ltd, which is subcontracted by Observational and Pragmatic Research Institute Pte Ltd, and OPRI) from UK National Health Service, British Lung Foundation, Aerocrine, AKL Ltd, Almirall, AstraZeneca, Boehringer Ingelheim, Chiesi, Eli Lilly, GlaxoSmithKline, Meda, Merck, Mundipharma, Napp, Novartis, Orion, Pfizer, Respiratory Effectiveness Group, Takeda, Teva Pharmaceuticals and Zentiva; payments for lectures/speaking from Almirall, AstraZeneca, Boehringer Ingelheim, Chiesi, Cipla, GlaxoSmithKline, Kyorin, Meda, Merck, Mundipharma, Novartis, Pfizer, Skyepharma, Takeda and Teva Pharmaceuticals; payment for manuscript preparation from Mundipharma and Teva Pharmaceuticals; patents (planned, pending or issued) from AKL Ltd; payment for the development of educational materials from GlaxoSmithKline and Novartis; stock/stock options from AKL Ltd which produces phytopharmaceuticals; owns $80 \%$ of Research in Real Life Ltd, which is subcontracted by OPRI, $75 \%$ of the social enterprise Optimum Patient Care Ltd and 75\% of OPRI; received payment for travel/accommodations/ meeting expenses from Aerocrine, Boehringer Ingelheim, Mundipharma, Napp, Novartis and Teva Pharmaceuticals; funding for patient enrolment or completion of research from Almirral, Chiesi, Teva Pharmaceuticals and Zentiva; and peer reviewer for grant committees of the Medical Research Council (2014), Efficacy and Mechanism Evaluation programme (2012), HTA (2014).

The other authors report no conflicts of interest in this work. 


\section{References}

1. Global Initiative for Chronic Obstructive Lung DG. Pocket Guide to COPD Diagnosis, Management and Prevention. Available from: http:// www.goldcopd.it/materiale/2015/GOLD_Pocket_2015.pdf. Accessed July $25,2016$.

2. Newman SP. Inhaler treatment options in COPD. Eur Respir Rev. 2005; 14:102-108.

3. Newman SP, Pavia D, Garland N, Clarke SW. Effects of various inhalation modes on the deposition of radioactive pressurized aerosols. Eur J Respir Dis Suppl. 1982;119:57-65.

4. Laube BL, Janssens HM, de Jongh FH, et al. What the pulmonary specialist should know about the new inhalation therapies. Eur Respir J. 2011;37(6):1308-1331.

5. Chrystyn $\mathrm{H}$. Is inhalation rate important for a dry powder inhaler? Using the In-Check Dial to identify these rates. Respir Med. 2003;97(2): 181-187.

6. Holmes MS, Seheult JN, O'Connell P, et al. An acoustic-based method to detect and quantify the effect of exhalation into a dry powder inhaler. J Aerosol Med Pulm Drug Deliv. 2015;28(4):247-253.

7. Al-Showair RA, Tarsin WY, Assi KH, Pearson SB, Chrystyn H. Can all patients with COPD use the correct inhalation flow with all inhalers and does training help? Respir Med. 2007;101(11):2395-2401.

8. Arora P, Kumar L, Vohra V, et al. Evaluating the technique of using inhalation device in COPD and bronchial asthma patients. Respir Med. 2014;108(7):992-998.

9. Broeders ME, Molema J, Hop WC, Folgering HT. Inhalation profiles in asthmatics and COPD patients: reproducibility and effect of instruction. J Aerosol Med. 2003;16(2):131-141.

10. Lavorini F, Levy ML, Corrigan C, Crompton G; ADMIT Working Group. The ADMIT series - issues in inhalation therapy. 6) Training tools for inhalation devices. Prim Care Respir J. 2010;19(4):335-341.

11. Melani AS, Bracci LS, Rossi M. Reduced peak inspiratory effort through the diskus $((\mathrm{R}))$ and the turbuhaler((R)) due to mishandling is common in clinical practice. Clin Drug Investig. 2005;25(8):543-549.

12. Price D, Bosnic-Anticevich S, Briggs A, et al; Inhaler Error Steering Committee. Inhaler competence in asthma: common errors, barriers to use and recommended solutions. Respir Med. 2013;107(1):37-46.

13. van der Palen J, Klein JJ, van Herwaarden CL, Zielhuis GA, Seydel ER. Multiple inhalers confuse asthma patients. Eur Respir J. 1999;14(5): 1034-1037.

14. Yu AP, Guerin A, de Leon DP, et al. Clinical and economic outcomes of multiple versus single long-acting inhalers in COPD. Respir Med. 2011;105(12):1861-1871.

15. Chrischilles E, Gilden D, Kubisiak J, Rubenstein L, Shah H. Delivery of ipratropium and albuterol combination therapy for chronic obstructive pulmonary disease: effectiveness of a two-in-one inhaler versus separate inhalers. Am J Manag Care. 2002;8(10):902-911.
16. Ketelaars CA, Schlosser MA, Mostert R, Huyer Abu-Saad H, Halfens RJ, Wouters EF. Determinants of health-related quality of life in patients with chronic obstructive pulmonary disease. Thorax. 1996;51(1): 39-43.

17. Stahl E, Lindberg A, Jansson SA, et al. Health-related quality of life is related to COPD disease severity. Health Qual Life Outcomes. 2005; 3:56.

18. Optimum Patient Care Research Database (OPCRD). Available from: http://optimumpatientcare.org/our-database/. Accessed July 25, 2016.

19. Roche N, Reddel H, Martin R, et al. Quality standards for real-world research. Focus on observational database studies of comparative effectiveness. Ann Am Thorac Soc. 2014;11(Suppl 2):S99-S104.

20. European Network of Centres for Pharmacoepidemiology and Pharmacovigilance. Available from: http://www.encepp.eu/. Accessed July 25, 2016.

21. GOLD. Gold Initiative for Chronic Obstructive Lung Disease. Available from: http://www.goldcopd.org/. Accessed July 25, 2016.

22. Price D, Chrystyn H, Kaplan A, et al. Effectiveness of same versus mixed asthma inhaler devices: a retrospective observational study in primary care. Allergy Asthma Immunol Res. 2012;4(4):184-191.

23. Melani AS, Bonavia M, Cilenti V, et al. Inhaler mishandling remains common in real life and is associated with reduced disease control. Respir Med. 2011;105(6):930-938.

24. Wieshammer S, Dreyhaupt J. Dry powder inhalers: which factors determine the frequency of handling errors? Respiration. 2008;75(1): $18-25$.

25. Yu AP, Guerin A, Ponce de Leon D, et al. Therapy persistence and adherence in patients with chronic obstructive pulmonary disease: multiple versus single long-acting maintenance inhalers. J Med Econ. 2011;14(4):486-496.

26. Punekar YS, Landis SH, Wurst K, Le H. Characteristics, disease burden and costs of COPD patients in the two years following initiation of longacting bronchodilators in UK primary care. Respir Res. 2015;16:141.

27. Price D, Bateman ED, Chisholm A, et al. Complementing the randomized controlled trial evidence base. Evolution not revolution. Ann Am Thorac Soc. 2014;11(Suppl 2):S92-S98.

28. Roy A, Battle K, Lurslurchachai L, Halm EA, Wisnivesky JP. Inhaler device, administration technique, and adherence to inhaled corticosteroids in patients with asthma. Prim Care Respir J. 2011;20(2): 148-154.

29. Broeders ME, Vincken W, Corbetta L; ADMIT Working Group. The ADMIT series - issues in inhalation therapy. 7. Ways to improve pharmacological management of COPD: the importance of inhaler choice and inhalation technique. Prim Care Respir J. 2011;20(3):338-343.

30. Caliskaner AZ, Ozturk C, Ceylan E, et al. The knowledge and considerations of the physicians regarding the inhaler devices in asthma and COPD: the INTEDA-1 study. Tuberk Toraks. 2013;61(3):183-192. 


\section{Supplementary materials}

Table SI Comorbidities, baseline co-medications and COPD classifications in each study cohort

\begin{tabular}{|c|c|c|c|}
\hline \multirow[t]{2}{*}{ Patient characteristics } & \multicolumn{2}{|l|}{ n (\%) } & \multirow[t]{2}{*}{$P$-value ${ }^{e}$} \\
\hline & $\begin{array}{l}\text { Similar-devices } \\
\text { cohort }(n=8,225)\end{array}$ & $\begin{array}{l}\text { Mixed-devices } \\
\text { cohort }(n=8,225)\end{array}$ & \\
\hline \multicolumn{4}{|l|}{ Comorbidities } \\
\hline Asthma ${ }^{\mathrm{a}}$ & $\mathrm{I}, 870(22.7)$ & $1,926(23.4)$ & 0.17 \\
\hline Asthma (active) $)^{a, c}$ & $896(10.9)$ & $896(10.9)$ & NA \\
\hline Diabetes $^{\mathrm{a}}$ & $1,388(16.9)$ & $1,356(16.5)$ & 0.487 \\
\hline Oral thrush ${ }^{\mathrm{b}}$ & $49(0.6)$ & $63(0.8)$ & 0.187 \\
\hline Rhinitis $^{\mathrm{a}}$ & $677(8.2)$ & $584(7.1)$ & 0.006 \\
\hline Rhinitis (active) ${ }^{b, d}$ & $345(4.2)$ & $331(4.0)$ & 0.579 \\
\hline Eczema $^{\mathrm{a}}$ & $1,162(14.1)$ & $1,143(13.9)$ & 0.665 \\
\hline Eczema (active) $)^{b, d}$ & $223(2.7)$ & $|8|(2.2)$ & 0.034 \\
\hline Cardiovascular disease $\mathrm{a}^{\mathrm{a}}$ & $2,811(34.2)$ & $2,817(34.3)$ & 0.918 \\
\hline Heart failure ${ }^{a}$ & $409(5.0)$ & 404 (4.9) & 0.918 \\
\hline Ischemic heart disease ${ }^{a}$ & I,693 (20.6) & $1,719(20.9)$ & 0.605 \\
\hline Hypertension ${ }^{\mathrm{b}}$ & $1,247(15.2)$ & $\mathrm{I}, 192(14.5)$ & 0.223 \\
\hline Gastroesophageal reflux disease ${ }^{a}$ & $586(7.1)$ & $586(7.1)$ & $>0.99$ \\
\hline GERD (active) $)^{b, d}$ & 400 (4.9) & $419(5.1)$ & 0.49 \\
\hline Osteoporosis ${ }^{\mathrm{a}}$ & $45 \mid(5.5)$ & $512(6.2)$ & 0.038 \\
\hline Pneumonia ${ }^{\mathrm{b}}$ & $75(0.9)$ & $113(1.4)$ & 0.006 \\
\hline Anxiety, depression & $373(4.5)$ & $381(4.6)$ & 0.763 \\
\hline \multicolumn{4}{|l|}{ Charlson comorbidity index score ${ }^{\mathrm{b}}$} \\
\hline 0 & $5,610(68.2)$ & $5,657(68.8)$ & 0.477 \\
\hline $1-4$ & $1,756(21.4)$ & $\mathrm{I}, 736(2 \mathrm{I} . \mathrm{I})$ & \\
\hline $5-9$ & $318(3.9)$ & $334(4.1)$ & \\
\hline$\geq 10$ & $54 \mid(6.6)$ & $498(6.1)$ & \\
\hline \multicolumn{4}{|l|}{ Co-medications } \\
\hline NSAIDs ${ }^{c}$ & $3,368(41.0)$ & $3,474(42.2)$ & 0.087 \\
\hline Paracetamol ${ }^{c}$ & $3,129(38.0)$ & $3,229(39.3)$ & 0.103 \\
\hline Beta blockers ${ }^{c}$ & $1,080(13.1)$ & $1,016(12.4)$ & 0.127 \\
\hline Statins ${ }^{c}$ & $2,518(30.6)$ & $2,640(32.1)$ & 0.027 \\
\hline \multicolumn{4}{|l|}{ Other measures of COPD severity } \\
\hline mMRC score (closest to index date) & & & $<0.00$ I \\
\hline Non-missing & $6,572(79.9)$ & $6,654(80.9)$ & \\
\hline $0-1$ & $3,863(58.8)$ & $3,765(56.6)$ & \\
\hline$\geq 2$ & $2,709(41.2)$ & $2,889(43.4)$ & \\
\hline GOLD grade (closest to index date) & & & Not tested \\
\hline Non-missing & $7,726(93.9)$ & $7,791(94.7)$ & \\
\hline$A$ & $717(9.3)$ & $717(9.2)$ & \\
\hline B & $4,796(62.1)$ & $4,788(61.5)$ & \\
\hline C & I,903 (24.6) & $1,918(24.6)$ & \\
\hline$D$ & $310(4.0)$ & $368(4.7)$ & \\
\hline
\end{tabular}

Notes: aWith a diagnostic code recorded at any time prior to or at the last extraction date; balculated for the baseline period including the last extraction date; ${ }^{\mathrm{c} a s t h m a}$

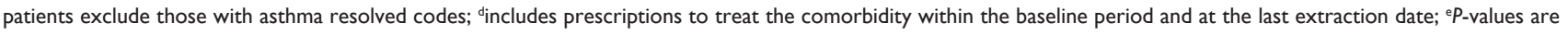
from conditional logistic regression models; GOLD grade was not compared between cohorts as there was a large amount of missing data.

Abbreviations: mMRC, modified Medical Research Council; GOLD, Global initiative for chronic Obstructive Lung Disease - groups based on 2015 GOLD Strategy; GERD, gastroesophageal reflux disease; NSAIDs, non-steroidal anti-inflammatory drugs; NA, not applicable (as variable was used for matching). 
Table S2 Combinations of COPD therapy during baseline year in each study cohort

\begin{tabular}{lll}
\hline Inhaler therapy & $\mathbf{n}(\%)$ & $\begin{array}{c}\text { Mixed-devices } \\
\text { cohort }(\mathbf{n = 8 , 2 2 5})\end{array}$ \\
\cline { 2 - 3 } & $\begin{array}{l}\text { Similar-devices } \\
\text { cohort }(\mathbf{n = 8 , 2 2 5})\end{array}$ & $3,775(45.9)$ \\
\hline SABA & $3,839(46.7)$ & $1,105(13.4)$ \\
SAMA + SABA & $1,075(13.1)$ & $209(2.5)$ \\
LABA \pm SAMA \pm SABA & $228(2.8)$ & $194(2.4)$ \\
LAMA \pm SAMA \pm SABA & $243(3.0)$ & $3(0.0)$ \\
LABA + LAMA \pm SAMA \pm SABA & $8(0.1)$ & $2,150(26.1)$ \\
ICS \pm SAMA \pm SABA & $2,271(27.6)$ & $757(9.2)$ \\
ICS + LABA \pm SAMA \pm SABA & $511(6.2)$ & $1(0.0)$ \\
ICS + LAMA \pm SAMA \pm SABA & $8(0.1)$ & $17(0.2)$ \\
LTRA \pm SAMA \pm SABA & $17(0.2)$ & $0(0.0)$ \\
LABA + LTRA \pm SAMA \pm SABA & $1(0.0)$ & $7(0.1)$ \\
ICS + LTRA \pm SAMA \pm SABA & $17(0.2)$ & $7(0.1)$ \\
ICS + LABA + LTRA \pm SAMA \pm SABA & $7(0.1)$ & \\
\hline
\end{tabular}

Abbreviations: SAMA, short-acting muscarinic agonist; SABA, short-acting beta agonist; LABA, long-acting beta agonist; LAMA, long-acting muscarinic antagonist; ICS, inhaled corticosteroid; LTRA, leukotriene receptor antagonist.

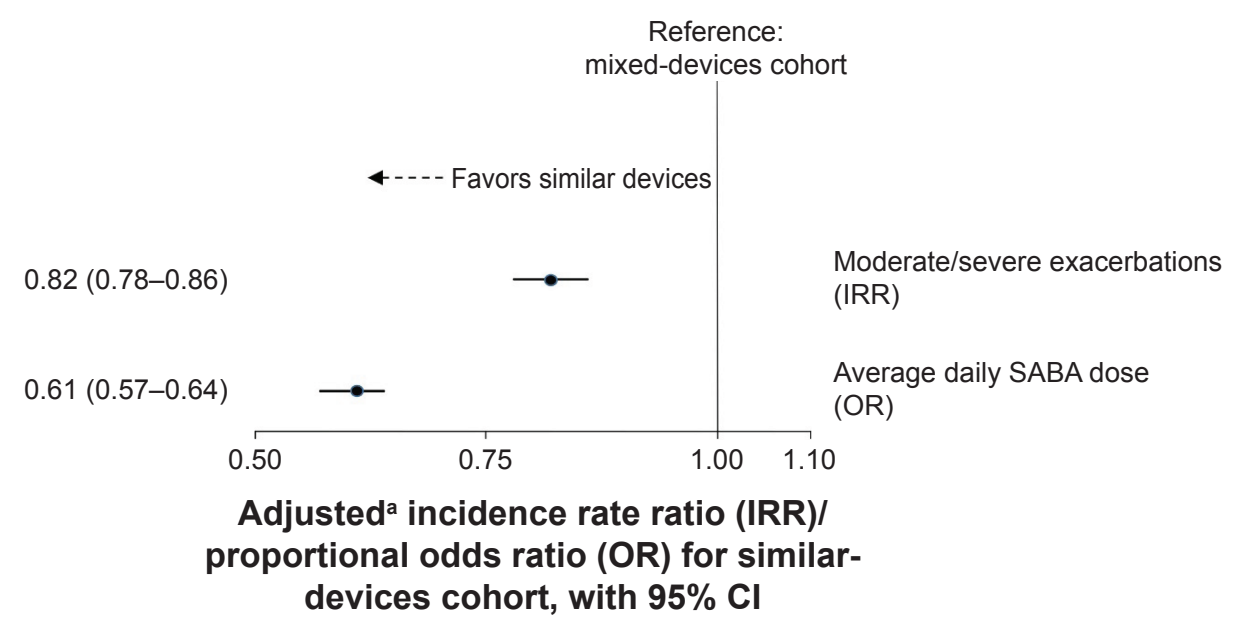

Figure SI Effect of similar versus mixed devices on primary and secondary COPD outcomes - alternative categorization of devices.

Notes: aIRR adjusted by COPD consultations, use of antibiotics, statins and paracetamol. OR adjusted by SABA dosage, use of statins, beta-blockers and paracetamol. $\mathrm{n}=7,545$ in each cohort, for this categorization of devices.

Abbreviations: $\mathrm{Cl}$, confidence interval; SABA, short-acting beta agonist.

Table S3 Combinations of COPD therapy during outcome year in each study cohort

\begin{tabular}{|c|c|c|}
\hline \multirow[t]{2}{*}{ Inhaler therapy } & \multicolumn{2}{|l|}{ n (\%) } \\
\hline & $\begin{array}{l}\text { Similar-devices } \\
\text { cohort }(n=8,225)\end{array}$ & $\begin{array}{l}\text { Mixed-devices } \\
\text { cohort }(n=8,225)\end{array}$ \\
\hline $\mathrm{LABA} \pm \mathrm{SAMA} \pm \mathrm{SABA}$ & $604(7.3)$ & $336(4.1)$ \\
\hline $\mathrm{LAMA} \pm \mathrm{SAMA} \pm \mathrm{SABA}$ & $47 \mathrm{I}(5.7)$ & $\mathrm{I}, 136(\mathrm{I} 3.8)$ \\
\hline $\mathrm{LABA}+\mathrm{LAMA} \pm \mathrm{SAMA} \pm \mathrm{SABA}$ & $51(0.6)$ & $250(3.0)$ \\
\hline$I C S \pm S A M A \pm S A B A$ & $2,464(30.0)$ & $653(7.9)$ \\
\hline$I C S+L A B A \pm S A M A \pm S A B A$ & $3,680(44.7)$ & $2,859(34.8)$ \\
\hline$I C S+L A M A \pm S A M A \pm S A B A$ & $90(I . I)$ & $498(6.1)$ \\
\hline$I C S+L A B A+L A M A \pm S A M A \pm S A B A$ & $780(9.5)$ & $2,358(28.7)$ \\
\hline $\mathrm{LABA}+\mathrm{LTRA} \pm \mathrm{SAMA} \pm \mathrm{SABA}$ & $0(0.0)$ & $2(0.0)$ \\
\hline LAMA + LTRA $\pm S A M A \pm S A B A$ & $4(0.1)$ & $3(0.0)$ \\
\hline $\mathrm{ICS}+\mathrm{LTRA} \pm \mathrm{SAMA} \pm \mathrm{SABA}$ & $12(0.2)$ & $3(0.0)$ \\
\hline $\mathrm{ICS}+\mathrm{LAMA}+\mathrm{LTRA} \pm \mathrm{SAMA} \pm \mathrm{SABA}$ & $4(0.1)$ & $3(0.0)$ \\
\hline$I C S+L A B A+L A M A+L T R A \pm S A M A \pm S A B A$ & II (0.I) & $48(0.6)$ \\
\hline ICS + LABA + LTRA $\pm S A M A+/ i S A B A$ & $53(0.6)$ & $76(0.9)$ \\
\hline Others & $\mathrm{I}(0.0)$ & $0(0.0)$ \\
\hline
\end{tabular}

Abbreviations: SAMA, short-acting muscarinic agonist; SABA, short-acting beta agonist; LABA, long-acting beta agonist; LAMA, long-acting muscarinic antagonist; ICS, inhaled corticosteroid; LTRA, leukotriene receptor antagonist. 


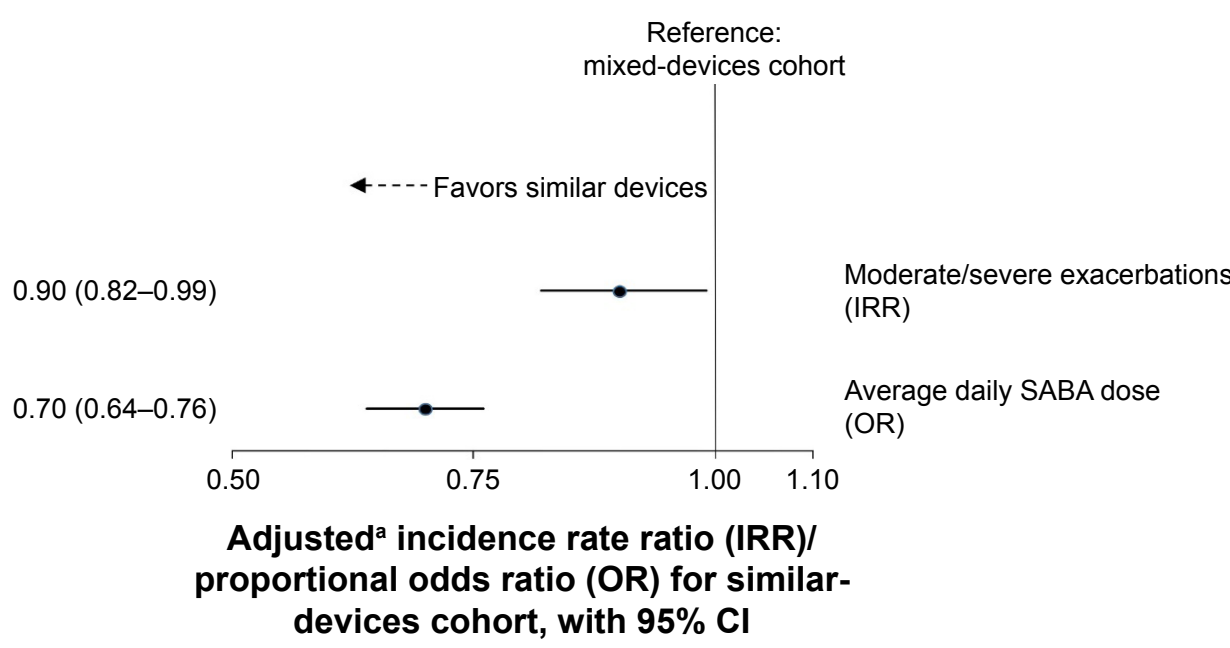

Figure S2 Effect of similar versus mixed devices on primary and secondary COPD outcomes - largest treatment combination subgroup.

Notes: aIRR adjusted by antibiotics courses, asthma diagnosis and use of paracetamol. OR adjusted by SABA dosage, use of statins, beta-blockers and paracetamol. $n=3,680$ (44.7\%) in similar-devices cohort and $n=2,859$ (34.8\%) in mixed-devices cohort.

Abbreviations: $\mathrm{Cl}$, confidence interval; SABA, short-acting beta agonist.

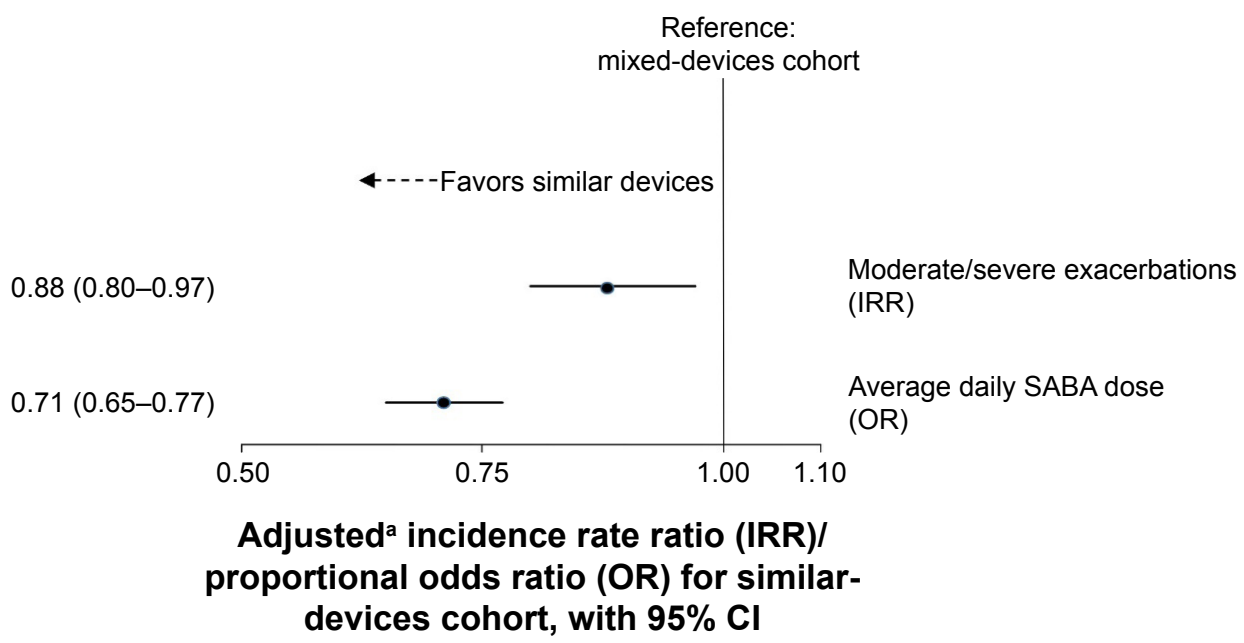

Figure S3 Effect of similar versus mixed devices on primary and secondary COPD outcomes - alternative categorization of devices and largest treatment combination subgroup.

Notes: alRR adjusted by antibiotics courses, asthma diagnosis and use of paracetamol. OR adjusted by SABA dosage, use of statins, beta-blockers and paracetamol. $n=3,762$ (49.9\%) in similar-devices cohort and $n=2,577$ (34.2\%) in mixed-devices cohort.

Abbreviations: $\mathrm{Cl}$, confidence interval; SABA, short-acting beta agonist.

International Journal of COPD

\section{Publish your work in this journal}

The International Journal of COPD is an international, peer-reviewed journal of therapeutics and pharmacology focusing on concise rapid reporting of clinical studies and reviews in COPD. Special focus is given to the pathophysiological processes underlying the disease, intervention programs, patient focused education, and self management protocols

\section{Dovepress}

This journal is indexed on PubMed Central, MedLine and CAS. The manuscript management system is completely online and includes a very quick and fair peer-review system, which is all easy to use. Visit $\mathrm{http}: / / \mathrm{www}$. dovepress.com/testimonials.php to read real quotes from published authors. 